\title{
Business Intelligence com Qlik Sense Aplicado ao Gerenciamento de Projetos em Scrum: um Estudo de Caso
}

\author{
Igor Castilho Valenciano ${ }^{1}$, Joyce Aline Oliveira ${ }^{1}$, Vanice Cunha ${ }^{2}$, Giulla Araújo ${ }^{3}$ \\ ${ }^{1}$ Faculdade de Engenharia - Universidade Federal de Mato Grosso (UFMT), Cuiabá - \\ MT - Brazil \\ ${ }^{2}$ Instituto de Computação - Universidade Federal de Mato Grosso (UFMT), Cuiabá - \\ MT - Brazil \\ ${ }^{3}$ Tribunal de Contas do Estado de Mato Grosso, Cuiabá - MT - Brazil \\ igorcvalenciano@gmail.com, Joyce.marins@ufmt.br, vanicedic.ufmt.br, \\ giullaetce.mt.gov.br
}

\begin{abstract}
The purpose of this article is to identify metrics to measure projects that make use of the SCRUM methodology and implement them in Qlik Sense, through the deduction of equations that represent them. A TCE-MT system was used as a case study to generate dashboards to monitor the implemented metrics. As a result, a preview of the team's performance in relation to the chosen system was obtained, based on the application of the metrics discussed in this article and data provided by the institution.

Resumo. O objetivo deste artigo é identificar métricas para mensurar projetos que fazem uso da metodologia SCRUM e implementá-las no Qlik Sense, inferindo equações que as representam. Um sistema do TCE-MT foi utilizado como estudo de caso para a geração de dashboards de acompanhamento das métricas implementadas. Como resultado obteve-se uma prévia do desempenho da equipe em relação ao sistema escolhido, baseado na aplicação das métricas discutidas neste artigo e dados fornecidos pela instituição.
\end{abstract}

\section{Introdução}

A metodologia SCRUM, é um framework baseado no empirismo e que busca ajudar pessoas, times e organizações a gerar valor através de soluções adaptáveis para problemas complexos [Schwaber e Sutherland 2020]. O SCRUM divide o projeto em ciclos denominados "sprint", também conhecido como iteração, que geralmente acontecem mensalmente. O ciclo define termos específicos para cada atividade ou função existente na operação, que é iniciada com a definição da prioridade dos itens que constam na lista de funcionalidades, conhecida como Product Backlog [Schwaber e Sutherland 2020].

Os métodos ágeis promovem um processo que exige um ciclo constante de inspeção, adaptação e melhoria [Sato 2007]. Bonfim (2013) afirma que é difícil medir se e quanto uma equipe está evoluindo ou quantificar outras informações sobre o seu desempenho e por conta disso, a maioria das equipes ágeis empregam o uso de métricas. Para Albino (2017), métricas podem ser qualitativas e quantitativas, devendo ser compreensíveis e utilizáveis com o intuito de promover ações de melhoria contínua, trazendo assim, visibilidade sobre a saúde do processo de desenvolvimento ao time. 
Um conjunto de métricas bem projetado além de permitir que os ciclos de desenvolvimento sejam constantemente inspecionados, adaptados e melhorados, pode ser utilizado para medir a qualidade dos produtos, dar suporte à tomada de decisão dos gerentes de projeto e aumentar o retorno de investimento do produto [Vicente 2010]. Budacu e Pocatilu (2018) ainda afirmam que utilizar métricas ágeis é mais eficiente do que mensurar produtividade através de linhas de código, permitindo o acompanhamento em tempo real da equipe de desenvolvimento.

De acordo com Phan e Vogel (2010), o cenário empresarial passou a ser cada vez mais competitivo conforme a aplicação de tecnologias de informação crescia na sociedade e dessa forma, o Business Intelligence (BI) é utilizado como um auxílio em gerenciamento de dados para tomadas de decisão e pesquisas de mercado. Gerenciamento de dados se refere ao processo de extrair, transformar e analisar os dados de uma organização ou negócio [Negash e Gray 2008].

Este artigo visa identificar métricas para mensurar projetos em SCRUM e implementá-las no Qlik Sense, que é uma ferramenta de BI. O trabalho é útil porque permite conhecer o uso e os benefícios de métricas, além da importância de combinar e exibir os dados obtidos em uma ferramenta de Business Intelligence. Desse modo, esta pesquisa difere dos trabalhos encontrados porque aborda a área de metodologia de desenvolvimento de software aplicado ao Qlik Sense e a revisão bibliográfica relacionada a métricas propostas por autores distintos.

\section{Metodologia}

Inicialmente foi realizado um estudo na literatura de métricas específicas para se gerenciar projetos baseados em metodologias ágeis, mais especificamente o SCRUM. As métricas encontradas foram implementadas no Qlik Sense. Para testar a implementação das métricas foi realizado um estudo de caso no sistema CONEX-E (Sistema de Controle Eletrônico Externo) do Tribunal de Contas do Estado de Mato Grosso (TCE-MT). O CONEX-E permite elaboração de relatórios técnicos e possui diversos módulos essenciais para as atividades do TCE-MT. No estudo, foi empregado o uso de uma pesquisa exploratória, cujo propósito é proporcionar maior familiaridade com o problema, com vistas a torná-lo mais explícito ou construir hipóteses [Gil 2002].

Os dados extraídos para o estudo de caso foram retirados do REDMINE, sistema de gerenciamento de projetos, usado pela equipe de desenvolvimento do TCE-MT. A extração manual desses dados visou organizá-los e ordená-los com o objetivo de gerar uma fonte de informação mensurável e passível de acompanhamento por meio de dashboards implementados no Qlik Sense, que consiste em uma ferramenta de BI de fácil utilização, pois aplica o conceito de self-service. De acordo com Affeldt e Junior (2013), a tecnologia BI, tem como objetivo melhorar a qualidade da informação entregue aos gestores, transformando dados em informações úteis que criam valor para a empresa.

Por limitação de dados, apenas as métricas (Tabela 1) de (1) indicador de bugs, (2) fator de complexidade da sprint, (3) agilidade do desenvolvimento, (4) quantidade de itens não previstos, (5) porcentagem de testes automatizados em relação aos testes manuais e (6) valor entregue na história de usuário foram mensuradas.

A implementação no Qlik Sense, além da importação de dados do CONEX-E, foi realizada definindo os itens mestre: Dimensão e Medida, fatores da ferramenta que representam a lista de sprints e as métricas, respectivamente. A Dimensão se refere ao 
agrupamento dos dados na visualização, representada pelo campo "lista de sprints" em todas as métricas implementadas. Com relação às Medidas, foram criadas definindo a equação inferida pertinente (Tabela 1) como expressão de valor desse tipo de item mestre, gerando resultados quantitativos.

\section{Resultados e discussão}

\subsection{Métricas para mensuração de projetos baseados em SCRUM}

Para que todo o ciclo proposto pelo SCRUM obtenha resultados satisfatórios, a mensuração auxilia no acompanhamento pontual do projeto, pois métricas são representações numéricas que determinam alguma característica de um software, projeto ou equipe. As métricas listadas na Tabela 1 foram encontradas na literatura e se destacaram porque apresentaram formas práticas, como equações, para medirem um determinado aspecto ou por terem evidenciado o uso dessas técnicas em um estudo de caso.

Tabela 1. Métricas disponibilizadas na literatura

\begin{tabular}{|c|c|}
\hline Autor & Equações inferidas \\
\hline RAM (2009) & $\begin{array}{l}\text { Fator de complexidade da sprint: } \sum \text { quantidade de módulos x que interagem } \\
\text { com interfaces de módulos y. }\end{array}$ \\
\hline JOHAN (2000) & $\begin{array}{l}\text { Indicador de bugs: } \sum \text { bugs que ocorreram na sprint. } \\
\text { Porcentagem de testes automatizados em relação aos manuais: } \\
\text { TA }=\text { (quantidade de testes automatizados } * 100) \text { / quantidade de testes manuais. }\end{array}$ \\
\hline SATO (2007) & $\begin{array}{l}\text { Funcionalidades testadas e entregues: } \mathrm{RTF}= \\
\text { (funcionalidades testadas e aceitas pelo cliente / quantidade total de } \\
\text { funcionalidades do projeto) } * 100 \\
\text { Fator de teste: } \\
\text { FT = quantidade de linhas de código de teste na iteração / quantidade de linhas } \\
\text { de produção na iteração }\end{array}$ \\
\hline $\begin{array}{l}\text { IKOMA et al } \\
(2009)\end{array}$ & $\begin{array}{l}\text { Agilidade do desenvolvimento: } \\
\mathrm{A}=\text { quantidade de funcionalidades validadas no período / quantidade média de } \\
\text { funcionalidades não validadas }\end{array}$ \\
\hline BONFIM (2013) & $\begin{array}{l}\text { Quantidade de itens não previstos: Qi }=\sum \text { itens não previstos no planejamento } \\
\text { Valor entregue na história de usuário: } \mathrm{VEi}=\sum \mathrm{Vi} \text { onde Vi é o valor entregue } \\
\text { naquela sprint, cujo parâmetro de avaliação é definido pelo Product Owner. }\end{array}$ \\
\hline
\end{tabular}

\subsection{Implementação no Qlik Sense}

O Qlik Sense permite conexões à fonte de dados externas para importar dados para o seu ambiente em nuvem de Data Analytics. Nesse estudo de caso, a importação foi de dados provenientes de um arquivo .xlsx, onde o Qlik Sense exibe um overview de suas 
informações importadas e permite aplicar edições, como a seleção de colunas da sua fonte de dados, possibilitando a exclusão ou modificação de um determinado conjunto.

Após finalizar o carregamento dos dados, a ferramenta exibe o dashboard de sua pasta, sendo possível construir a visualização dos seus dados para o usuário. Para a construção de gráficos ou outro componente visual, é necessário definir os itens mestres dimensão e medida que irão compor o gráfico. No dashboard implementado, foram utilizados os componentes: indicador do tipo KPI (Key Performance Indicators), indicador do tipo mostrador, gráfico de barras, gráfico de linhas e gráfico de pizza. Portanto, a implementação das 6 métricas quantitativas elencadas na tabela 1 foi feita considerando 16 sprints do CONEX-E, no período compreendido entre 9 de novembro de 2020 e 27 de agosto de 2021. Nesse intervalo, empregando a métrica de indicador de bugs, através de um somatório, foram quantificados 52 bugs, resultado considerável visto que corresponde a mais de um terço do Product Backlog, composto de 132 itens.

Com relação à métrica de fator de complexidade da sprint, a equação inferida define que quanto maior a interação entre módulos distintos, maior é o seu resultado, que evidenciou o sucesso no planejamento das sprints, visto que a carga de complexidade foi bem distribuída e em algumas delas não houve interação entre módulos do sistema (Figura 1). Ainda, no levantamento realizado, a métrica de quantidade de itens não previstos identificou apenas 1 item não planejado no Product Backlog, resultado baixo e provavelmente motivado pela carência de informações no sistema de gerenciamento de projetos, pois ao considerar 16 sprints, seria normal que surgissem mais itens não previstos ao longo do processo.

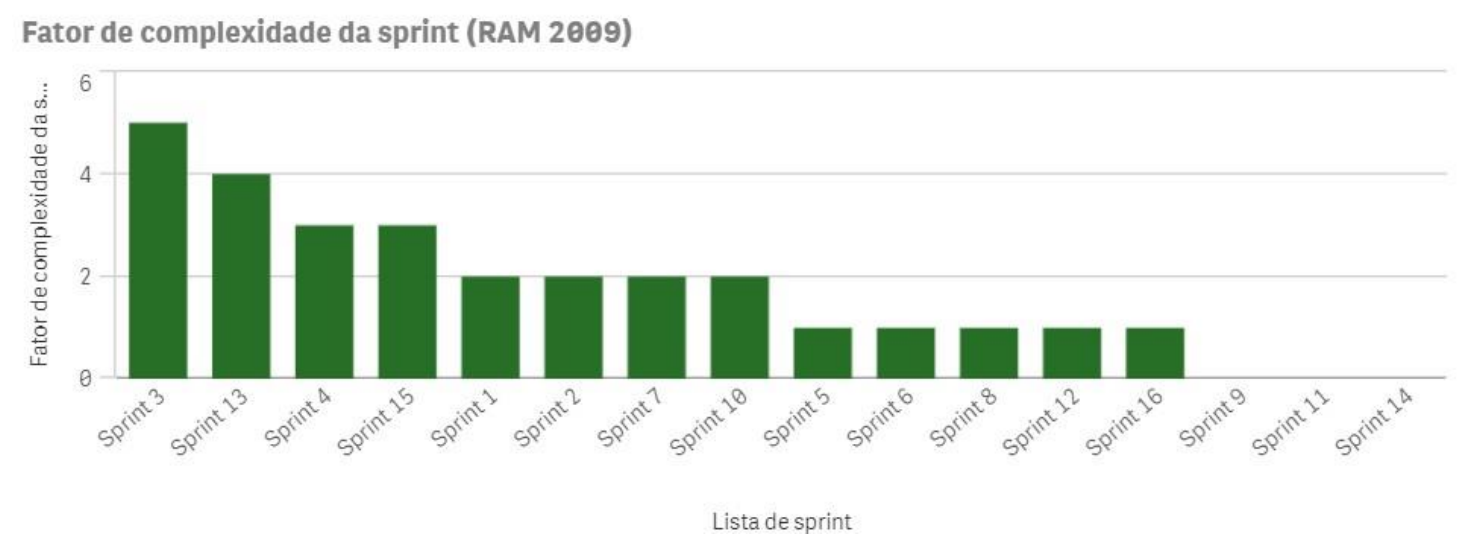

Figura 1. Métrica de fator de complexidade da sprint, onde no eixo $x$ são apresentadas as sprints e no eixo y são apresentados os fatores de complexidade de cada sprint.

Contudo, a métrica de agilidade do desenvolvimento, que relaciona a quantidade de itens validados e a quantidade média dos itens do Product Backlog, se manteve estável, mesmo quando houve uma grande quantidade de bugs e que poderia causar atrasos e diminuir a agilidade. A linha do gráfico dessa métrica teve um leve aumento apenas nas últimas sprints, resultado esperado pois demonstra a proximidade do término do conjunto de dados extraído (Figura 2). Referente ao valor entregue na história de usuário, considerando que Bonfim (2013) define que esse cálculo é feito pelo Product Owner, nesse estudo de caso o parâmetro adotado foi a relação entre os itens da sprint e a quantidade de bugs, ou seja, quanto menor a quantidade de erros, maior foi o valor entregue na história de usuário e consequentemente, ocupou uma fatia maior do gráfico, 
como observado na Figura 3. Sendo assim, é visível que poucas sprints obtiveram um considerável valor entregue na história de usuário, comprovando a necessidade de uma intervenção no processo de desenvolvimento para conter a quantidade de erros.

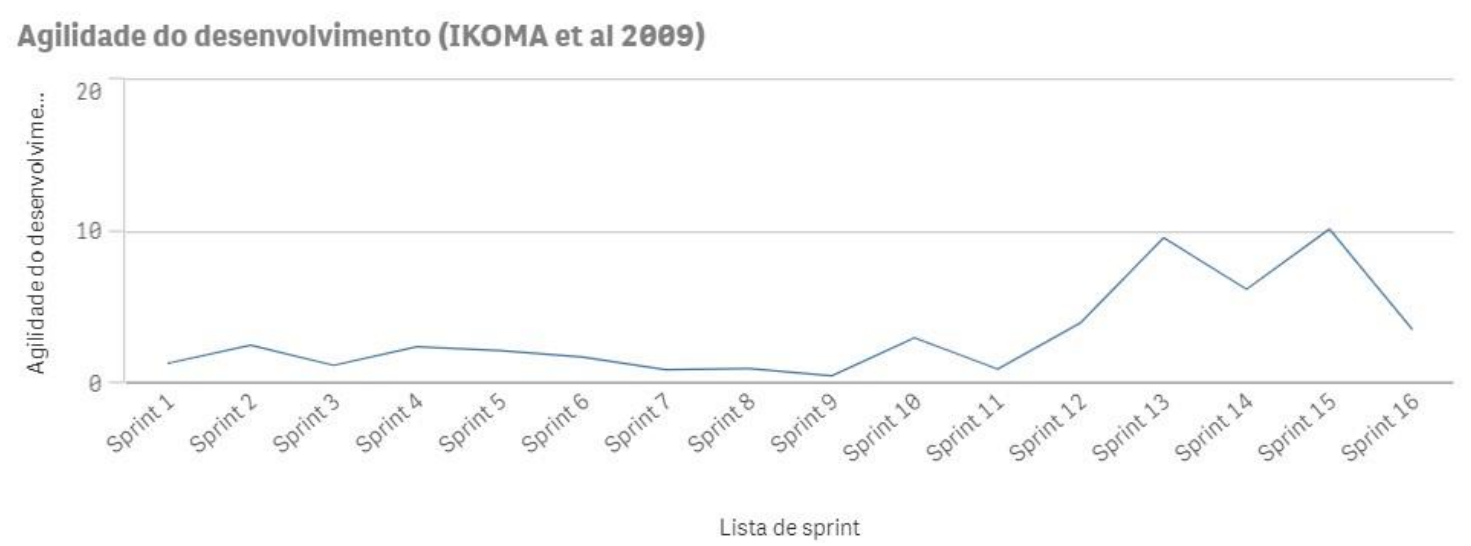

Figura 2. Métrica de agilidade do desenvolvimento, onde no eixo $x$ são apresentadas as sprints e no eixo y são apresentados os valores de agilidade no desenvolvimento de cada sprint.

Valor entregue na história de usuário (BONFIM 2013)

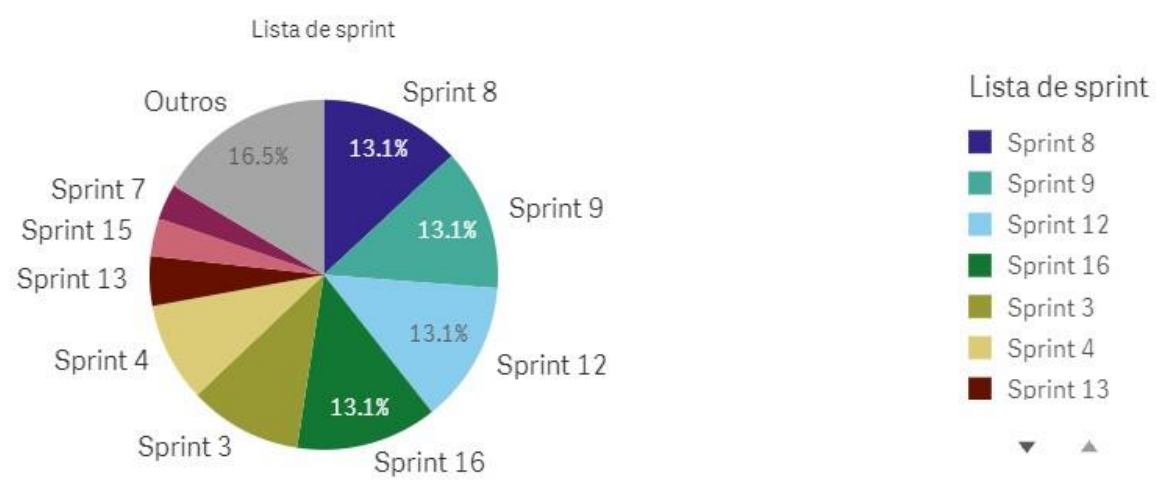

Figura 3. Métrica de valor entregue na história de usuário

Com o objetivo de visualizar os fatores que contribuem para um determinado resultado, o Qlik Sense foi utilizado para combinar o uso de duas métricas para plotar um gráfico, como é visto na Figura 4 que evidencia a relação de bugs e itens da sprint com a métrica de valor entregue na história de usuário, mesclando um gráfico de barras e de linhas. Por fim, devido o TCE-MT empregar apenas o uso de testes manuais, a métrica de porcentagem de testes automatizados em relação aos manuais se manteve zerada, resultando em um cenário passível de grandes quantidades de erros como evidenciado no indicador de bugs. Verificou-se, portanto, que a instituição precisa adotar a execução de testes automatizados, ou melhorar o processo de testes manuais para diminuir erros e aumentar a qualidade do software entregue [Bernardo e Kon 2008]. 


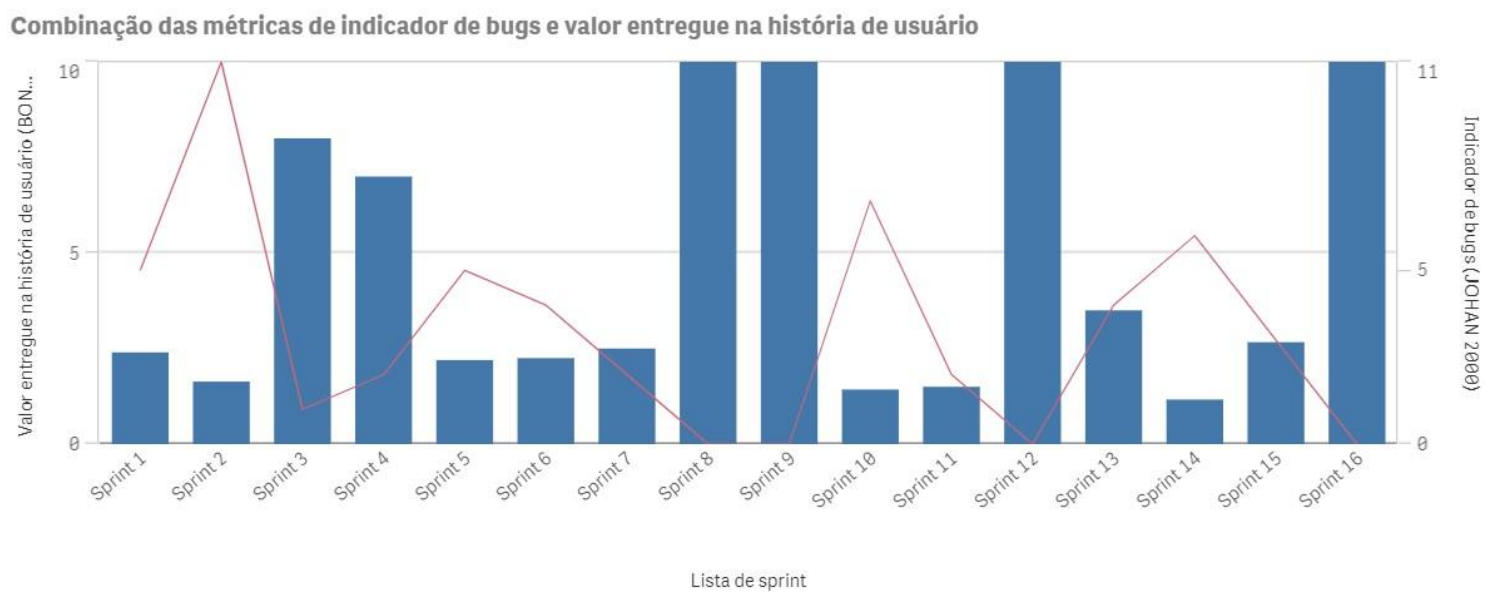

Figura 4. Gráfico de combinação entre as métricas indicador de bugs e valor entregue na história de usuário, onde no eixo x são apresentadas as sprints, sendo que as barras representam o valor entregue e a linha em vermelho se refere ao indicador de bugs.

Após a finalização da implementação das métricas no Qlik Sense, os resultados foram apresentados aos líderes de equipes de desenvolvimento do TCE-MT, que identificaram a necessidade de acompanhamento pontual não só do CONEX-E, mas de todos os projetos baseados em SCRUM. Os líderes também perceberam a necessidade de corrigir falhas nos testes de software, a necessidade de alimentar o REDMINE com a documentação completa sugerida pelo SCRUM e de alimentar o REDMINE com todos os dados das sprints referentes a cada métrica implementada. Cabe enfatizar, que o estudo está em andamento e mais métricas serão implementadas, de acordo com o feedback dos líderes.

\section{Conclusão}

Este trabalho apresentou a aplicação de métricas ágeis no sistema CONEX-E do TCEMT por meio de extração de dados do REDMINE referente a dezesseis sprints. Essa fonte de informação foi aplicada em equações inferidas a partir de artigos da literatura que abordam a definição de métricas ágeis. Com as informações obtidas, foi possível identificar as principais deficiências da área de desenvolvimento do TCE-MT referente ao CONEX-E, como por exemplo, a ausência de testes automatizados, a necessidade de melhoria dos testes manuais e a detecção de um número considerável de bugs. As limitações do trabalho consistem na escassez de informações no REDMINE, visto que as equipes utilizam o SCRUM há pouco tempo e a cultura de cadastrar os dados dos projetos baseados nessa metodologia ágil ainda está sendo fomentada. Como continuidade da pesquisa, serão feitas novas extrações de dados e implementações de mais métricas, esperando-se como resultado que o dashboard de métricas no Qlik Sense auxilie, avalie e monitore os projetos SCRUM da instituição, aumentando os índices de produtividade e eficiência. $\mathrm{O}$ estudo é útil para organizações que visam mensurar projetos baseados em SCRUM e consiste em uma relevante contribuição visto que poucos artigos mostram como métricas para acompanhamento de projetos ágeis podem ser implementadas em uma ferramenta de BI. 


\section{Referências}

Affeldt, F. S., Junior, S. D. S. (2013) "Information architecture analysis using business intelligence tools based on the information needs of executives", JISTEM - Journal of Information Systems and Technology Management, São Paulo.

Albino, Raphael D. (2017) "Métricas Ágeis - Obtenha melhores resultados em sua equipe”, Casa do Código, ISBN: 978-85-5519-276-0.

Bernardo, Paulo C., Kon, Fabio. (2008) “A Importância dos Testes Automatizados", Engenharia de Software Magazine, páginas 54-57.

Bonfim, M. (2013) “A importância das métricas para equipes agéis", http://www.devmedia.com.br/a-importancia-das-metricas-paraequipesageis/28542\#ixzz2idwKEJhi, Agosto.

Budacu, Eduard N., Pocatilu, Paul. (2018) "Real Time Agile Metrics for Measuring Team Performance", Informatica Economica vol. 22, páginas 70-79.

Gil, A. C. (2002) "Como elaborar projetos de pesquisa”, 4.ed. São Paulo: Atlas

Ikoma, M.; Ooshima, M.; Tanida,T.; Oba, M.; Sakai, S. (2009) "Using a Validation Model to Measure the Agility of Software Development in a Large Software Development Organization", https://ieeexplore.ieee.org/abstract/document/5070967, Agosto.

Johan, P. (2000) "Quantitative Approach for Lightweight Agile Process Assessment", http://citeseerx.ist.psu.edu/viewdoc/download?doi=10.1.1.609.210\&rep=rep1\&type= pdf, Agosto.

Negash, Solomon., Gray, Paul. (2008) "Business Intelligence. In: Handbook on Decision Support Systems 2", International Handbooks Information System. Springer, Berlin, Heidelberg.

Phan, Dien D., Vogel, Doug. (2010) “A model of customer relationship management and business intelligence systems for catalogue and online retailers", Information \& Management, Vol.47, páginas 69-77.

Ram, P. (2009) "Agile Metrics: A seminal approach for calculating Metrics in Agile Projects", https://www.projectsmart.co.uk/agile-metrics-a-seminal-approachforcalculating-metrics-in-agile-projects.php, Agosto.

Sato, D. T. (2007) "Uso eficaz de métricas em métodos ágeis de desenvolvimento de software”, Dissertação de Mestrado, Instituto de Matemática e Estatística (USP), São Paulo, Brasil.

Schwaber, K., Sutherland, J. (2020) "Guia do Scrum - Um guia definitivo para o Scrum: As regras do jogo", https://scrumguides.org/docs/scrumguide/v2020/2020ScrumGuide-PortugueseBR.pdf, Agosto.

Vicente, André Abe. (2010) "Definição e gerenciamento de métricas de teste no contexto de métodos ágeis", https://www.teses.usp.br/teses/disponiveis/55/55134/tde23062010083439/publico/AndreVicente.pdf, Março. 